\title{
Revisión breve sobre la nebulización en tiempos de COVID-19
}

\author{
Brief review on nebulization in times of COVID-19
}

\author{
Désirée Larenas-Linnemann,* Ramsés Hernández Pliego, ${ }^{\ddagger}$ Jorge García Magaña ${ }^{\S}$ \\ * Directora del Centro de Excelencia en Asma y Alergia en el Hospital Médica Sur. \\ ‡ Doctorado en Investigación en Medicina. Therapeutic Area Lead Respiratory \& Immunology Franchise. \\ $\S$ Clinical Science Liason.
}

\section{RESUMEN}

\begin{abstract}
El asma, una enfermedad bronquial crónica, cursa con exacerbaciones que ameritan manejo inmediato con tratamiento inhalado que frecuentemente se aplica a través de nebulizaciones. Sin embargo, la preocupación actual por la transmisión aérea del SARS-CoV-2 ha llevado a brindar tratamiento subóptimo a los pacientes que sufren una crisis asmática por el temor al contagio. Se revisa el tema y se proponen recomendaciones sustentadas en la literatura.
\end{abstract}

Palabras clave: Asma; COVID-19, niños, SARS-CoV-2.

\section{INTRODUCCIÓN}

\section{Asma}

El asma es una enfermedad inflamatoria crónica heterogénea que afecta los bronquios ocasionando obstrucción por contracción espasmódica de la vía aérea inferior con episodios recurrentes de disnea paroxística que se manifiestan por sibilancias, disnea, opresión torácica y tos. ${ }^{1}$ Se acompaña de limitación variable del flujo aéreo respiratorio. Si el ataque es grave, puede llegar a presentarse insuficiencia respiratoria o hasta ser fatal en caso de no controlarse..$^{2,3}$

El asma afecta de 1 a $8 \%$ de la población en el mundo. ${ }^{3}$ En México la prevalencia en adultos se ha reportado en hombres en $3.3 \%$ y en mujeres

\section{ABSTRACT}

Asthma, a chronic bronchial disease, presents with exacerbations that merit immediate management with inhaled treatment that is frequently applied through nebulisations. However, the current concern about the airborne transmission of SARS-CoV-2 has led to suboptimal treatment for patients suffering an asthma attack due to fear of contagion. The subject is reviewed and recommendations based on the literature are provided.

Keywords: Asthma, COVID-19, children, SARS-CoV-2.

en $6.2 \%$; y en niños y adolescentes, según la metodología ISAAC (International Study of Asthma and Allergies in Childhood), en $17.0 \%$ en niños y en $19.2 \%$ en adolescentes. ${ }^{2}$ Desafortunadamente, la fracción de los pacientes con enfermedad controlada es muy baja (9.4\%), mientras que $55.8 \%$ tiene asma parcialmente controlada y $34.8 \%$ tiene asma no controlada; uno de los retos en el manejo del asma es mejorar estas cifras. ${ }^{4}$ Así, en México en el periodo de 2010 a 2018, el asma fue la causa primaria de hospitalización en 87,726 pacientes admitidos en hospitales públicos, y la causa primaria de muerte en $12,495 .{ }^{2}$ La mortalidad total fue de $10.41 / 100,000$ habitantes, con una tasa promedio anual de $1.16 \mathrm{y}$ una diferencia de género para el grupo femenino de 44.9 y $53.0 \%$ para los grupos de edad $<15$ años y $>15$, respectivamente. ${ }^{2}$

Citar como: Larenas-Linnemann D, Hernández PR, García MJ. Revisión breve sobre la nebulización en tiempos de COVID-19. Rev Latin Infect Pediatr. 2021; 34 (3): 135-141. https://dx.doi.org/10.35366/102236

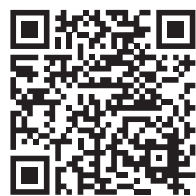




\section{Corticosteroides inhalados}

Los corticosteroides inhalados son la primera línea de tratamiento para preescolares con asma o sibilancias recurrentes. Son la opción preferida en niños con desencadenantes múltiples de sibilancias y síntomas frecuentes, especialmente aquéllos con eosinofilia en sangre y sensibilidad a alérgenos aéreos. ${ }^{5}$

Una revisión sistemática de 29 ensayos clínicos controlados aleatorizados (ECA) en 3,592 preescolares demostró que los esteroides inhalados redujeron la frecuencia de episodios de sibilancias independientemente del fenotipo y atopia. ${ }^{6}$ Del mismo modo, un metaanálisis más reciente de 15 estudios con un total de 3,278 niños de seis años con asma recurrente reveló que la dosis media diaria de esteroides inhalados redujo el riesgo de exacerbaciones en $30 \%$ en comparación con placebo. Además, cuando el análisis se realizó en el subgrupo de niños con asma persistente, el riesgo de exacerbación se redujo en $44 \%$.

En un reciente estudio, Wu y colaboradores compararon la eficacia y seguridad del tratamiento escalonado de fluticasona nebulizada con el tratamiento escalonado de budesónida nebulizada en el asma infantil para prevenir recaídas. La población (con edades de hasta dos años) se dividió en dos grupos, propionato de fluticasona $250 \mu \mathrm{g}$ dos veces al día por seis semanas y luego $125 \mu \mathrm{g}$ dos veces al día por seis semanas $(n=389)$ y budesónida $500 \mu \mathrm{g}$ dos veces al día por seis semanas seguidos de $250 \mu \mathrm{g}$ dos veces al día por seis semanas $(\mathrm{n}=$ 389) suministrados a través de un nebulizador jet. Los pacientes en el grupo budesónida tuvieron un periodo mayor libre de síntomas postratamiento (165 días vs. 112 días, $p<0.0001)$ que los pacientes del grupo fluticasona. ${ }^{8}$ Así, la evidencia publicada de los beneficios de los esteroides inhalados en niños preescolares con sibilancias es cada vez más sólida.

\section{CONCEPTOS DE NEBULIZACIÓN}

Un aerosol médico es cualquier combinación de partículas líquidas o sólidas de un medicamento suspendidas en un gas portador. ${ }^{9}$ El tamaño de las partículas depende de la solución (densidad, viscosidad y tensión superficial) así como de la velocidad y el flujo del gas que pasa por la fase líquida o sólida para generar partículas en suspensión. Un aumento de la velocidad del gas disminuye el tamaño de las partículas (nebulizar de 6 a $8 \mathrm{~L} / \mathrm{min}$ ). ${ }^{10}$
Los aerosoles médicos pueden administrarse a través de nebulizadores, inhaladores presurizados de dosis medida ( $\mathrm{pMDI}$ ) o inhaladores de polvo seco (DPI). ${ }^{9}$

El nebulizador se define como el dispositivo que puede convertir un líquido en gotitas de aerosol adecuadas para la inhalación por parte del paciente (nebulización), en particular cuando es el propio paciente el que coloca el medicamento en el dispositivo antes de cada tratamiento. Los nebulizadores neumáticos, jet o de chorro de aire son los más utilizados, aunque los nebulizadores ultrasónicos son cada vez más comunes. ${ }^{11}$

Aun cuando la terapia con aerosoles es un procedimiento fundamental y rutinario en el tratamiento de enfermedades pulmonares en el hogar y en entornos de atención médica, tiene un potencial de emisiones fugitivas durante la terapia debido a la generación de aerosoles y gotitas como fuente de patógenos respiratorios. La emisión fugitiva se define como aerosoles que se han liberado del dispositivo de aerosol durante la espiración del paciente, también los aerosoles médicos no inhalados por el paciente, pero que pasan a la atmósfera. El tamaño de las partículas de las emisiones fugitivas varía de 0.860 a $1.437 \mu \mathrm{m}$ y hasta $50 \%$ del aerosol generado durante la terapia permanece en el aire en el ambiente interior durante varias horas. ${ }^{12}$

\section{Ventajas y desventajas de la nebulización}

En las Guías de la Estrategia Global para el Manejo y Prevención del Asma 2020 (GINA 2020) se considera el tratamiento nebulizado en los niños de cinco años o menores. Después de los IDM los nebulizadores son los únicos sistemas alternativos de suministro de medicamentos inhalados viables en niños pequeños; se reservan para los niños preescolares y los que no aprenden a emplear un dispositivo espaciador. Es relevante recordar que si se usa un nebulizador para suministrar esteroides, es aconsejable emplear una boquilla para evitar su contacto con los ojos en niños que cooperen. ${ }^{3}$

El tratamiento nebulizado forma parte integral del manejo de la vía aérea tanto durante una crisis asmática como en otras patologías que se acompañan de inflamación bronquial o broncoconstricción como la bronquiolitis y las sibilancias inducidas por infecciones virales en la infancia. ${ }^{13}$

Los nebulizadores liberan el medicamento a dosis terapéuticas en aproximadamente 15 minutos con una distribución pulmonar efectiva en pacientes 
Rev Latin Infect Pediatr. 2021; 34 (3): 135-141

de cualquier edad. La terapia nebulizada, con una boquilla cuando se considere necesaria, se considera confiable, fácil de usar, eficaz y eficiente, especialmente en los jóvenes pacientes con crisis asmática. ${ }^{13}$

Las desventajas comunes de los nebulizadores a menudo incluyen falta de portabilidad, complejidad de su configuración, costo, prolongados tiempos de entrega de dosis altas de medicamentos y eficiencia persistentemente baja de administración pulmonar. ${ }^{13,14}$

Sin embargo, en los últimos años ha evolucionado la administración de fármacos nebulizados. Los nebulizadores se volvieron más portátiles, más ligeros, más silenciosos y con uso de pilas. Las tecnologías de respiración mejorada y activadas por la respiración optimizaron la eficiencia de los nebulizadores de chorro al reducir la pérdida de medicamento al medio ambiente y la exposición de los cuidadores. Desarrollos recientes, la malla vibratoria y las tecnologías de administración de aerosoles adaptables proporcionan dosis precisas y reproducibles; reducen las pérdidas de aerosol durante la espiración, y permiten un depósito más profundo de aerosol en los pulmones, junto con una duración de tratamiento más corta. También pueden dar retroalimentación al paciente y al cuidador que administró la dosis prescrita. ${ }^{15}$

\section{Nebulizaciones durante la pandemia, precauciones}

En el contexto de la presente pandemia de COVID19 se requiere extremar las medidas de control de transmisión de infecciones. Los nebulizadores generan altos volúmenes de partículas de aerosol de 1-5 $\mu \mathrm{m}$ de diámetro que pueden transportar virus y bacterias a nivel alveolar, además de alcanzar una gran distancia en un patrón natural de dispersión. Adicionalmente, estas partículas pueden estimular la tos del paciente y de las personas alrededor, aumentando el riesgo de contagio, por lo que existe la posibilidad de transmisión de coronavirus viables a personas sanas, incluyendo al personal de salud. ${ }^{16}$

La Organización Mundial de la Salud (OMS) y los Centros para el Control y la Prevención de Enfermedades (CDC, por sus siglas en inglés) recomiendan tomar precauciones con las partículas de aerosol y el contacto con todos los pacientes sospechosos o diagnosticados con COVID-19. Las precauciones de transmisión aérea de agentes infecciosos deben aplicarse al realizar procedimientos médicos generadores de aerosoles (PMGA) como la aspiración abierta de secreciones respiratorias, intubación, broncoscopia y reanimación cardiopulmonar. La información obtenida durante la pandemia sugiere que la nebulización de medicación también es un PMGA. ${ }^{17}$

En la sección correspondiente a medidas preventivas de las guías GINA 2020 relativas a COVID-19 se indica que, en la medida de lo posible, se evite el uso de nebulizadores debido al riesgo de dispersión viral a otros pacientes y a trabajadores de la salud. Los nebulizadores pueden esparcir partículas virales respiratorias hasta un metro de distancia. ${ }^{3}$

De igual forma, en la Guía Española para el Manejo del asma (GEMA) versión 5.0 se señala, con evidencia proveniente de estudios no aleatorizados, observacionales o no controlados, que en el tratamiento de los pacientes con asma y COVID-19 no se empleen nebulizadores para la aerosolización de fármacos, (sino dispositivos acoplados a espaciadores o cámaras de inhalación) ni equipos de ventilación no invasiva de una sola rama y sin filtro bacteriano ubicado antes del puerto de salida. ${ }^{18}$

El Consenso Internacional sobre COVID-19 de Neumólogos recomendó: a) evitar el uso de medicamentos nebulizados en pacientes sospechosos/diagnosticados con COVID-19 debido a un mayor riesgo de aerosolización que llevará a una posible transmisión del SARS-CoV-2; b) reservar el tratamiento con broncodilatadores nebulizados para el broncoespasmo agudo; c) usar inhaladores de dosis medidas con espaciador para el manejo de condiciones crónicas; d) evitar la inducción de esputo con solución salina hipertónica nebulizada; y e) si se inicia la terapia nebulizada, los pacientes deben mantenerse en habitación aislada con presión negativa con todas las precauciones de control de infecciones transmitidas por el aire. ${ }^{19}$

\section{Nebulizaciones durante la pandemia: las recomendaciones más actualizadas}

Sin embargo, los lineamientos más recientes de expertos respiratorios del Reino Unido señalan que durante la nebulización, el aerosol proveniente de un dispositivo (líquido de la cámara del nebulizador) no porta partículas virales derivadas del paciente. Si una partícula de aerosol impacta con una membrana mucosa contaminada, ésta deja de ser transportada en el aire y por lo tanto, no forma parte del aerosol. El personal de salud debe emplear la higiene apropiada de manos cuando ayude a los pacientes a retirar nebulizadores y mascarillas de oxígeno. ${ }^{20}$ En particular, el lineamiento sobre COVID-19 en asma grave sugiere que los pacientes continúen 
empleando sus nebulizadores. Esto porque en un paciente con neumonía por SARS-CoV-2 moderadagrave la capacidad para inhalar está reducida y con ello el depósito del medicamento inhalado por un IDM-espaciador o en polvo seco es bajo. Al mismo tiempo, el aerosol del líquido de la cámara del nebulizador no porta partículas virales del paciente. ${ }^{21}$

Recientemente, un grupo de expertos hindúes recomienda que los pacientes con asma continúen empleando sus esteroides inhalados como siempre, pero se eviten hasta donde sea posible las nebulizaciones, empleando en su lugar los inhaladores de dosis medidas con espaciador. Indican que las nebulizaciones se usen si no hay opción o riesgo de muerte por asma. En tales casos recomiendan los nebulizadores accionados por la respiración, boquillas en lugar de mascarillas y filtros o válvulas unidireccionales, siempre que sea posible. ${ }^{22}$

Levin y colaboradores recomiendan que cuando se requieran las nebulizaciones: ${ }^{23}$

1. Se apliquen en habitaciones aisladas con presión negativa.

2. El personal de salud vista equipo de protección personal que incluya mascarilla N95.

3. En asma que amenaza la vida ( $\geq 1$ criterio: $\mathrm{SpO}_{2}$ $<80 \%$, cianosis, flujo espiratorio máximo $<33 \%$, esfuerzo respiratorio débil, tórax "silencioso", bradicardia, arritmia, hipotensión arterial, agotamiento, confusión, coma).

Más recientemente, Sethi y colaboradores consolidan las recomendaciones de medidas protectoras para tratamiento nebulizado dependiendo del sitio donde se proporcionen: ${ }^{24}$

Hogar:

1. Evitar nebulización en presencia de otros.

2. Higiene estricta del nebulizador.

3. Realizar la nebulización cerca de ventanas abiertas / áreas de mayor circulación de aire.

Hospital:

1. Adherencia a los lineamientos estrictos de sanitización.

2. Uso de equipo de protección personal en presencia de pacientes infecciosos.

3. Uso de habitaciones con presión negativa.

4. Desechar/desinfectar el equipo de protección personal después de cada uso.
5. Mantener una distancia de al menos dos metros entre pacientes.

Uso de filtros con nebulizadores.

Consolidando las anteriores recomendaciones, Ari sugiere 12 recomendaciones cuando se necesiten nebulizaciones en pacientes con COVID-19 leve (Tabla 1). ${ }^{12}$

Considerando que las recomendaciones no están sustentadas en evidencia científica sólida, al tomar una decisión informada sobre si nebulizar puede ser un manejo adecuado en su paciente, sugerimos tomar en cuenta estos factores:

1. Eficacia: ¿La terapia con un inhalador de dosis medida y espaciador (con mascarilla) ofrece una alternativa igualmente buena para el paciente en este momento?

a. En pacientes graves nebulizar puede ser más eficaz y permite la administración de tratamiento paralelo como oxígeno suplementario

b. En niños pequeños, nebulizar posiblemente permite que penetre más medicamento a nivel bronquial.

\section{Seguridad:}

a. ¿Existe un riesgo de contagio del personal que realiza la nebulización?

a.1. En área de urgencia de hospital: sí.

a.2. En cuarto de hospital, con medidas estrictas de aislamiento del paciente, buena ventilación y equipo de protección personal del personal: mínimo.

a.3. En consultorio con paciente con síntomas bronquiales: posiblemente sí (todo paciente puede ser portador asintomático).

a.4. En domicilio, paciente con COVID: aplica lo mismo que en hospital (2.1.b).

a.5. En domicilio, paciente con asma/sibilancias: mínimo.

b. ¿Existe un riesgo para el paciente si no recibe el tratamiento óptimo?

b.1. Paciente con asma controlada: poco.

b.2. Paciente con asma exacerbada/sibilancias: sí.

b.3. Paciente con COVID-19 grave: sí. 
Rev Latin Infect Pediatr. 2021; 34 (3): 135-141

Tabla 1: Estrategias prácticas para nebulizaciones en pacientes con COVID-19 leve.

1. Evite la administración innecesaria de medicamentos en aerosol a pacientes con COVID-19

2. Use esteroides inhalados para prevenir el empeoramiento del asma y los ataques de asma

3. Utilice pMDI o DPI para la administración de medicamentos en aerosol en lugar de nebulizadores si su paciente está despierto y puede realizar patrones de respiración específicos

4. Considere usar nebulizadores con boquilla o cánula nasal de alto flujo si el inhalador aumenta la tos o si el paciente tiene insuficiencia respiratoria aguda

5. Coloque filtros en los nebulizadores antes de administrar medicamentos en aerosol a los pacientes. Utilice filtros HEPA si es posible

6. No use una mascarilla con nebulizadores

7. Prefiera usar una boquilla con nebulizadores de chorro y malla

8. Conecte filtros o válvulas unidireccionales a la tubuladura de gran diámetro del nebulizador de chorro para prevenir emisiones fugitivas durante la terapia de aerosol

9. Agregue un filtro al otro extremo de la boquilla para eliminar la liberación de aerosoles al medio ambiente cuando utilice un nebulizador de malla

10. Administre nebulizaciones en habitaciones con presión negativa

11. Use equipo de protección personal, incluida mascarilla N95, gafas/careta, doble guante, bata o delantal si la bata no es resistente a los líquidos

12. Considere utilizar vigilancia a distancia (telemedicina) para evaluar a los pacientes infectados por coronavirus en su hogar y minimizar el uso de las instalaciones sanitarias

De la mano de los dos casos descritos a continuación, esperamos aclarar todo este concepto un poco más.

Caso 1. «Clara» de dos y medio año nuevamente presenta tos que duró toda la noche, vomitó en cuatro ocasiones flemas hialinas y contenido gástrico. La madre comenta que al inicio de la semana tuvo un poco de fiebre y gripa, pero desde antier inició con tos progresiva. Ésta ya es la tercera vez este año que se le complica una gripa. La madre igualmente presentó una leve gripa hace ocho días; ya está asintomática. Han guardado bien el aislamiento físico durante la pandemia, "Clara» sólo sale al jugar a su patio personal de la casa con su hermano mayor, pero sin tener contacto con otros niños. En relación al tratamiento: siguió sus indicaciones de darle formoterol + budesónida 80/4.5 $\mu$ g dos inhalaciones cada 12 horas con su espaciador Vortex ${ }^{\circledR}$. La respuesta fue parcial y llora al darle el inhalador. En la exploración se aprecia tos, disnea, sibilancias en las bases pulmonares, leve tiraje intercostal y subcostal.

\section{¿Prescribiría nebulizaciones?}

\section{Mi sugerencia: sí.}

Argumentación: I. Eficacia: la alternativa, el inhalador presurizado de dosis medida (IDMp) con espaciador parece no servirle, menos cuando llora con ello. II. Seguridad: riesgo bajo. La familia ya ha estado expuesta a los aerosoles que "Clara» genera al toser. Además, es poco probable que lo que presenta sea COVID-19 porque ya ha presentado cuadros semejantes recientemente, no hay claras situaciones de posible contagio y los síntomas son poco sugestivos de COVID-19, tanto los de ella como los de familiares. (Advertencia: pero nunca se puede descartar por completo).

¿Con qué nebulizaría? Broncodilatador y budesónida, dosis altas (de 0.5 a $1 \mathrm{mg}$ cada 12 horas) por siete días. ${ }^{25,26}$

¿Daría tratamiento de mantenimiento por unas semanas más? Primero investigaré su índice predictivo del asma (IPA), qué indica en niños de cero a tres años con sibilancias frecuentes, qué tan probable es que desarrollen asma a los cinco años.

"Clara» tenía dermatitis atópica desde bebé y usted investiga si tiene alergias pidiendo lgE específica en sangre contra ácaro, gato, perro, y los hongos Alternaria y Cladosporium: es positiva para ácaro de polvo casero y Alternaria. Con ello tiene el IPA positivo y mayor riesgo de desarrollar asma en un futuro y con ello seguir con recaídas como presenta hoy. Entonces sí sugeriría seguir en las noches con una nebulización por dos a tres meses para reducir recaídas de sibilancias con infecciones del tracto respiratorio alto, sobre todo en temporadas difíciles durante el año, aunque la evidencia para tal manejo aún no es sólida. ${ }^{26,27}$

Caso 2. «Pedro» de tres años tiene rinitis y asma alérgicas por alergia a varios tipos de polen. Se hospitalizó hace cinco días por crisis asmática después de una gripa. El manejo fue con corticosteroide sistémico por cinco días y nebulizaciones con broncodilatador. Fue dado de alta por mejoría.

\section{¿Prescribiría nebulizaciones en casa?}

Mi sugerencia: si no sabe cooperar bien con IDMpespaciador, sí nebulizar corticosteroide. 
Argumentación: Eficacia: si no coopera con IDMp-espaciador, mejor nebulizar. Seguridad: riesgo para familiares es bajo, a no ser que se haya sobreinfectado con COVID-19 en el hospital. El riesgo para «Pedro» sí es elevado si no recibe tratamiento adecuado. Después de una crisis asmática se tiene que seguir con manejo con esteroide inhalado estrictamente por mínimo 12 meses porque durante este tiempo existe mayor riesgo de recaída y nueva hospitalización. ${ }^{3,28}$ Cada crisis asmática daña al pulmón. Ahora depende de si «Pedro» ya sabe usar bien IDMp y espaciador + mascarilla. Si no coopera bien con este manejo, recomiendo dar el corticosteroide inhalado por nebulización.

¿Con qué nebulizaría? Corticosteroide para nebulizar dosis media, por ejemplo, budesónida $500 \mu \mathrm{g}$.

¿Daría tratamiento de mantenimiento por meses? Sí, se dará en las noches por 12 meses o hasta que aprenda a usar el IDMp-espaciadormascarilla.

Comentario: en niños pequeños ( $<30$ meses) con asma grave se ha demostrado que el manejo con budesónida nebulizado, $1 \mathrm{mg}$ cada 12 horas por 12 semanas redujo la frecuencia de crisis asmáticas de 83 a $40 \%$ y redujo el tiempo de exposición a corticosteroide oral; este efecto persistió aún tres meses después de haber terminado el tratamiento. ${ }^{29}$

Caso 3. «Juan» de 48 años fue diagnosticado con COVID-19 hace nueve días, inició con tos al quinto día, la cual se manejó con Symbicort $160 \mu \mathrm{g}$ : dos inhalaciones cada 12 horas. Presenta neumonía de focos múltiples en una tomografía de ayer. Siente leve opresión del pecho, sin disnea. Satura $82 \%$. Usted decide agregar oxigenoterapia al resto del manejo.

\section{En cuanto al manejo respiratorio: ¿prescribiría nebulizaciones?}

Mi sugerencia: sí, procurando cuidar el riesgo de contagio al nebulizar.

Argumentación: Eficacia: en este paciente grave es mejor nebulizar. Seguridad: el riesgo de contagio puede ser aceptable, siempre y cuando se procure aislar al paciente durante el tiempo que se esté nebulizando y ventilar bien después, al igual que uso de cubrebocas N95, careta, bata y cuidado de manos (guantes o gel) (domicilio o cuarto hospital). En urgencias: riesgo elevado de contagio para personal médico.
Comentario: en pacientes con neumonía por SARS-CoV-2, el corticosteroide inhalado forma parte de las indicaciones médicas. Como ya se explicó, se prefiere la administración con inhalador de dosis medida y espaciador cuando haya personal médico cerca que podría infectarse. Sin embargo, en un ambiente con aislamiento del paciente (un cuarto separado con buena ventilación en domicilio o un cuarto de aislamiento en hospital), nebulizar puede ser preferible porque aumenta la cantidad de medicamento que se logra administrar a las vías aéreas periféricas y además se puede combinar el esteroide con un broncodilatador en una misma sesión de nebulización.

¿Con qué nebulizaría? Broncodilatador y corticosteroide dosis media, por ejemplo, budesónida $0.250 \mathrm{mg}, 1.5 \mathrm{~mL}$ tres veces al día.

¿Por cuánto tiempo nebulizaría? Mientras permanezca con insuficiencia respiratoria. Ya que se logre destetar del oxígeno, se puede cambiar por IDMp-espaciador por unas semanas más. En COVID19 existe un alto grado de inflamación y daño pulmonar incipiente si no se controla la inflamación desde el inicio de síntomas pulmonares con manejo tópico. Hay que tener muy claro que en la primera semana de síntomas, cuando aún hay virus muy activo, usar corticosteroide sistémico puede propiciar la multiplicación viral y acelerar un desenlace fatal en la experiencia de la que subscribe. Los corticosteroides sistémicos se deben reservar para los casos avanzados graves en la segunda semana de la evolución. ${ }^{30}$

\section{CONCLUSIÓN}

La terapia nebulizada es una alternativa para el tratamiento de asma potencialmente fatal en niños menores de cinco años. Se brindan criterios de valoración y recomendaciones de acuerdo a los sitios donde se administre la terapia nebulizada. Es un campo que aún requiere mayor investigación.

\section{REFERENCIAS}

1. Larenas-Linnemann D et al. Manejo integral del asma. 2021. En prensa.

2. Carrillo G, Mendez-Domínguez N, Datta-Banik R, FigueroaLopez F, Estrella-Chan B, Alvarez-Baeza A et al. Asthma mortality and hospitalizations in Mexico from 2010 to 2018: Retrospective epidemiologic profile. Int J Environ Res Public Health. 2020; 17 (14): 5071.

3. Global Initiative for Asthma. Global Strategy for Asthma Management and Prevention, 2020. Accessed 01 January 2021] Available in: www.ginasthma.org. 
Rev Latin Infect Pediatr. 2021; 34 (3): 135-141

4. Solé D, Aranda CS, Wandalsen GF. Asthma: epidemiology of disease control in Latin America - short review. Asthma Res Pract. 2017; 3: 4.

5. Fainardi V, Santoro A, Caffarelli C. Preschool wheezing: trajectories and long-term treatment. Front Pediatr. 2020; 8: 240.

6. Castro-Rodriguez JA, Rodrigo GJ. Efficacy of inhaled corticosteroids in infants and preschoolers with recurrent wheezing and asthma: a systematic review with metaanalysis. Pediatrics. 2009; 123: e519-525.

7. Kaiser SV, Huynh T, Bacharier LB, Rosenthal JL, Bakel LA, Parkin PC et al. Preventing exacerbations in preschoolers with recurrent wheeze: a meta-analysis. Pediatrics. 2016; 137: e20154496.

8. Wu Z, Bian X, Hui L, Zhang J. Nebulized step-down budesonide vs. fluticasone in infantile asthma: $A$ retrospective cohort study. Exp Ther Med. 2020; 19 (3): 1665-1672.

9. The science of aerosol drug delivery. En: Douglas S. Gardenhire, editor. A guide to aerosol delivery devices for respiratory therapists. $4^{\text {th }}$ ed. USA: American Association for Respiratory Care; 2017, pp. 1-8.

10. Área de asma de SEPAR; Área de enfermería de SEPAR; Departamento de asma ALAT. Consenso SEPAR-ALAT sobre terapia inhalada. Arch Bronconeumol. 2013; 49 (Suppl 1): 2-14.

11. Boe J, Dennis JH, O'Driscoll BR, Bauer TT, Carone M, Dautzenberg B et al. European Respiratory Society Task Force on the use of nebulizers. European Respiratory Society Guidelines on the use of nebulizers. Eur Respir J. 2001; 18 (1): 228-242.

12. Ari A. Practical strategies for a safe and effective delivery of aerosolized medications to patients with COVID-19. Respir Med. 2020; 167: 105987

13. Murphy KR, Hong JG, Wandalsen G, Larenas-Linnemann D, El Beleidy A, Zaytseva OV et al. Nebulized inhaled corticosteroids in asthma treatment in children 5 years or younger: a systematic review and global expert analysis. $J$ Allergy Clin Immunol Pract. 2020; 8 (6): 1815-1827.

14. Longest W, Spence B, Hindle M. Devices for improved delivery of nebulized pharmaceutical aerosols to the lungs. $J$ Aerosol Med Pulm Drug Deliv. 2019; 32 (5): 317-339.

15. Araújo ADD, Correia-de-Sousa J. COPD: Will there be room for nebulisers after the current COVID-19 pandemic? Open Respiratory Archives 2020; 2 (4): 265-266.

16. Amirav I, Newhouse MT. Transmission of coronavirus by nebulizer: a serious, underappreciated risk. CMAJ. 2020; 192 (13): E346.

17. Benge CD, Barwise JA. Aerosolization of COVID-19 and contamination risks during respiratory treatments. Fed Pract. 2020; 37 (4): 160-163.

18. Sociedad Española de Neumología y Cirugía Torácica. GEMA 5.0. Guía española para el manejo del asma. [Consulta 29 Diciembre 2020] Disponible en: https://semg. es/images/documentos/GEMA 5.0.pdf.

19. Treatment options for COVID-19. En: Joseph T, editor. International Pulmonologist's Consensus on COVID-19. 2nd. ed. India: 2020, pp. 34-43.

20. Gobierno del Reino Unido. COVID-19: Guidance for the remobilisation of services within health and care settings. Infection prevention and control recommendations. [Accessed 02 January 2021] Available in: https://assets. publishing.service.gov.uk/government/uploads/system/
uploads/attachment_data/file/910885/COVID-19_Infection prevention_and_control_guidance_FINAL_PDF_20082020. pdf.

21. NICE. COVID-19 rapid guideline: severe asthma. [Accessed 02 January 2021] Available in: https://www.nice.org.uk/ guidance/ng166.

22. Banothu KK, Bhat JI, Das RR, Dhochak N, Ghimire JJ, Goyal JP et al. Expert opinion on restoration of pediatric pulmonology services during the SARS-CoV-2 pandemic. Indian Pediatr 2020; 57 (11): 1055-1059.

23. Levin M, Ansotegui IJ, Bernstein J, Chang YS, Chikhladze $M$, Ebisawa $\mathrm{M}$ et al. Acute asthma management during SARS-CoV-2-pandemic 2020. World Allergy Organ J. 2020; 13 (5): 100125.

24. Sethi S, Barjaktarevic IZ, Tashkin DP. The use of nebulized pharmacotherapies during the COVID-19 pandemic. Ther Adv Respir Dis. 2020; 14: 1753466620954366.

25. Bacharier LB, Phillips BR, Zeiger RS, Szefler SJ, Martinez FD, Lemanske RF Jr et al. Episodic use of an inhaled corticosteroid or leukotriene receptor antagonist in preschool children with moderate-to-severe intermittent wheezing. J Allergy Clin Immunol. 2008; 122 (6): 1127-1135 e8.

26. Zeiger RS, Mauger D, Bacharier LB, Guilbert TW, Martinez FD, Lemanske RF Jr et al. Daily or intermittent budesonide in preschool children with recurrent wheezing. N Engl J Med. 2011; 365 (21): 1990-2001.

27. Volovitz B, Soferman R, Blau H, Nussinovitch M, Varsano I. Rapid induction of clinical response with a short-term highdose starting schedule of budesonide nebulizing suspension in young children with recurrent wheezing episodes. J Allergy Clin Immunol. 1998; 101 (4 Pt 1): 464-469.

28. Larenas-Linnemann D, Salas-Hernandez J, Vazquez-Garcia JC, Ortiz-Aldana I, Fernandez-Vega M, Del Rio-Navarro BE et al. Mexican Asthma Guidelines: GUIMA 2017. Revista Alergia México. 2017; 64 Suppl 1: s11-s128.

29. De Blic J, Delacourt C, Le Bourgeois M, Mahut B, Ostinelli $\mathrm{J}$, Caswell $\mathrm{C}$ et al. Efficacy of nebulized budesonide in treatment of severe infantile asthma: a double-blind study. J Allergy Clin Immunol. 1996; 98 (1): 14-20.

30. Wiersinga WJ, Rhodes A, Cheng AC, Peacock SJ, Prescott HC. Pathophysiology, transmission, diagnosis, and treatment of coronavirus disease 2019 (COVID-19): a review. JAMA. 2020; 324 (8): 782-793.

Financiamiento: AstraZeneca.

Conflicto de intereses: Los autores declaran haber recibido financiamiento de AstraZeneca.

Cláusula de exención de responsabilidad: Las opiniones expresadas en el artículo son propias de los autores y no constituyen una posición oficial de las instituciones en que laboran o de la institución financiadora.

Correspondencia:

Dra. Désirée E.S. Larenas-Linnemann

Hospital Médica Sur, Torre II, Cons. 602

Puente de Piedra 150,

Col. Toriello-Guerra, 14050,

Alcaldía Tlalpan, Ciudad de México.

E-mail: marlar1@prodigy.net.mx 